Send your letters to the Editor,

British Dental Journal,

64 Wimpole Street

London

W1G 8YS

Email bdj@bda.org

Priority will be given to letters less than 500 words long.

Authors must sign the letter, which

may be edited for reasons of space.

\section{HOT POTATO TOPIC}

Sir, Roberts et al. (BDJ 2008: 204: E7) describe a simple method of dental age assessment which they claim provides a rapid and accurate estimate of age in children and emerging adults. This topic is now a hot potato politically, as the Home Office UK Border Agency is keen to apply the method to estimate the ages of asylum seekers, to decide whether they are a child or an adult based on a cut-off of 18 years. In the light of this political sensitivity the robustness of the method needs examining.

The method uses a previously assembled database of children whose ages and tooth stages are known. For a new subject, each of their teeth in turn is associated with the mean age of database children with the same tooth stage, and these 'tooth' ages are averaged across the child's teeth to estimate their chronological age. Roberts et al. show that the resulting mean age has a narrow confidence interval (CI) and hence high precision. However, what they do not say is that this CI applies to the population mean age, not to the age of the index child. To obtain the CI for the child, which is the more relevant statistic than the mean, and which is up to five times wider than the population mean CI, requires an additional calculation which they do not discuss. Thus the precision claimed for the method is exaggerated.

Another weakness of the method is its inability to handle mature stage $\mathrm{H}$ teeth. A mean age cannot be ascribed to such teeth (as the age range is unbounded upwards), so they are simply omitted from the calculation. As a result the estimate of the child's age is biased downwards. For example a young woman with 13 teeth at stage $\mathrm{H}$ and three at stage $G$ has her age estimated from just the stage $\mathrm{G}$ teeth, ignoring the evidence of her many mature teeth.

A third and related problem with the method relates to the phrase 'emerging adult' in the title. Once children are over 16 years or so, all their teeth bar the third molars are mature and hence ineligible. Thus for emerging adults the ageing relies on the third molars, of which there are between none and four (as against 16 for a younger child), and as a result the precision is much reduced. Stages $\mathrm{G}$ and $\mathrm{H}$ together bracket the age of 18 years, stage $\mathrm{G}$ having a mean age of 17.5 years and an associated $95 \% \mathrm{CI}$ of \pm 2.8 years, with stage $\mathrm{H}$ somewhat later. Yet stage $\mathrm{H}$ can be seen in emerging adults as young as 15 years, while stage $G$ is seen as late as 23 years. Thus the age range of stages $G$ and $H$ is too wide to provide useful evidence of an individual being under or over 18 years.

In summary, the methodological weaknesses of the method described by Roberts et al. make it unsuitable for assessment of dental age in individual children, and particularly in emerging adults.

\section{T. J. Cole, London}

The authors, Graham Roberts, Aviva Petrie and Victoria Lucas, respond: We thank Professor Cole for his interest in our paper and respond to his comments in order. We share some of his concerns and ask him and your readers to be aware that this is 'work in progress'. We draw attention to the section in our Discussion which stated that 'other investigators are encouraged to repeat and extend this work to determine whether there is a general applicability of the method'. We also stated that there will be ongoing research aimed at extending and improving the method.

Professor Cole pointed out that we omit- ted to say that the confidence interval for the population mean age is substantially narrower than the relevant interval for the age of an individual child. We apologise for any misinterpretation of the results that may have arisen as a consequence. Readers should be reassured that we recognised this shortly after the publication of the paper and so the confidence interval for the mean has not been used in practice in relation to an individual child's estimate for some time. Furthermore, there is work in progress to provide appropriate measures of precision which relate to the estimated age of a particular child.

Professor Cole's letter does not make any reference to the section entitled 'Testing the method for accuracy'. We remind readers that in this section we applied our method by reusing, with blind assessment, the radiographs of 50 patients of known chronological age. These cases did not form part of the database. We found that the maximum likely difference between chronological age and our estimated dental age was 1.65 years and, on average, the DAA overestimated the age by 0.29 years.

This brings into question Professor Cole's comment that the omission of Stage $H$ biases age assessment downwards. Because tooth growth is an ordered process, genetically controlled, the omission of Stage $H$ will not influence the estimation of age from the remaining teeth which have not yet completed growth. As an extreme example, consider the LL6 which, as determined by our current database, will finish its growth (ie reach Stage $H$ ) at the latest by 11.07 years. Therefore using only age information on a LL8 at Stage $G$, which has a minimum age of 14.41 years, will not underestimate dental age. It is interesting to note that Professor Cole, in effect, 
supports not using Stage $H$ by stating in the penultimate paragraph of his letter that 'once children are over 16 years or so, all their teeth bar the third molars are mature and therefore ineligible'.

We agree with Professor Cole that the age ranges of Stages $G$ and $H$ of third molars make it difficult to provide useful evidence of an individual being under or over 18 years of age. This has led to further work in this contentious area which enables us to estimate the probability that an individual is over 18 years of age. For example, the probability of an individual being over 18 years of age with LL8G is $26.6 \%$ or with LL8H is $67.0 \%$.

We are grateful to Professor Cole for his attention to detail. Such a critical approach can only lead to improved reliability of age estimates. It is important to recognise that, to date, our approach has provided one of the most accurate estimates of age. This is supported by the excellent results obtained when we submitted our DAA method to a 'blind' test of subjects of known chronological age. No other investigator has submitted their results to such robust scrutiny. Our database is available for any investigator who wishes to further his/her research on the subject: please contact the first author on graham.j.roberts@kcl.ac.uk.

Declaration of interests. Graham Roberts uses income from the DAA clinic at King's College Hospital to fund research in this area. He also has a pecuniary interest in this funding. He is co-supervisor, with Professor Cole, of Susan Parekh's PhD. Victoria Lucas conducts research that is funded by the DAA income and also has a small pecuniary interest in the DAA income. Aviva Petrie has no competing interests to declare.

DOI: 10.1038/sj.bdj.2008.1030

\section{CALCULUS OR TUMOUR?}

Sir, we would like to share with your readers an unusual case of a patient with dental calculus mimicking an oral tumour. An 82-year-old Caucasian woman was referred for diagnosis of an abnormal extensive hard mass in the mouth. Her past medical history included poorly controlled hypertension and type-2 diabetes. The patient was complaining about a growing tumour mass causing swelling and pain in the mandibular region. Examination showed an apparently edentulous mouth with a hard tissue formation of $4 \mathrm{~cm}$ covering the left side of the mandibular anterior alveolar ridge extending over the facial and lingual gingiva (Fig. 1). Radiographs revealed deposits completely covering all surfaces of two remaining teeth (Fig. 2). The diagnosis of dental calculus was made and the teeth were extracted. One week later the patient showed complete healing of the alveolus.

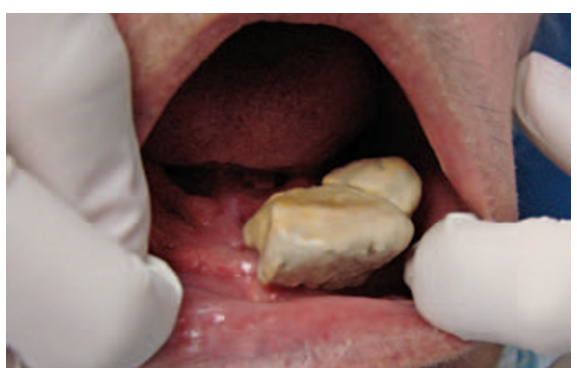

Fig. 1 A hard tissue mass covering the left side of mandibular anterior alveolar ridge

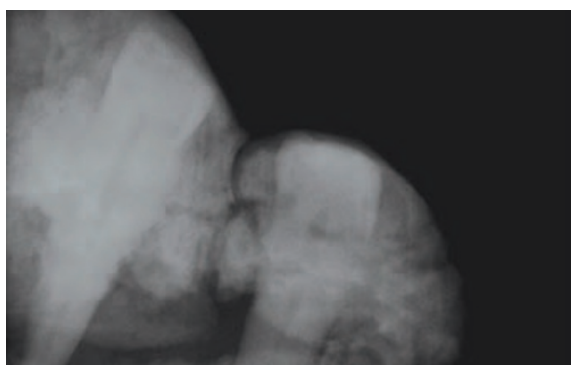

Fig. 2 Radiograph evidence of two remaining teeth inside the hard mass

Diabetes mellitus can lead to marked dysfunction of the secretory capacity of the salivary glands and saliva may be associated with the distribution pattern of supragingival calculus. Dental calculus is mineralised dental plaque permeated with crystals of various calcium phosphates and diabetic patients have an increased salivary calcium and protein concentration compared to non-diabetics. ${ }^{1}$ Additional studies have shown that various serum antioxidants are decreased in diabetes mellitus patients, including superoxide dismutase (SOD), ascorbic acid (vitamin C), uric acid (UA), and glutathione. ${ }^{2}$

Although an association between poorly controlled type 2 diabetes mellitus and severe periodontitis has been well documented, ${ }^{3}$ this clinical presentation is unusual and may have led to unnecessary procedures. A large dental calculus deposit has been previously suspected to be a neoplastic lesion because of its morphological features and exams such as three-dimensional computed tomography, scintigraphy, and pathological biopsy were taken to diagnose the condition. ${ }^{4}$

\section{K. L. Ortega, A. C. Luiz, F. M. Martins}

São Paulo

1. Mata A D, Marques D, Rocha S et al. Effects of diabetes mellitus on salivary secretion and its composition in the human. Mol Cell Biochem 2004; 261: 137-142.

2. Zloczower M, Reznick A Z, Zouby R 0, Nagler R M. Relationship of flow rate, uric acid, peroxidase, and superoxide dismutase activity levels with complications in diabetic patients: can saliva be used to diagnose diabetes? Antioxid Redox Signal 2007; 9: 765-773.

3. Tsai C, Hayes C, Taylor G W. Glycemic control of type 2 diabetes and severe periodontal disease in the US adult population. Community Dent Oral Epidemiol 2002; 30: 182-192.

4. Minoru M, Akinori I, Hitomi S, Yumiko O, Shun'ichiro N. A case of a giant dental calculus suspected to be a neoplastic lesion. Jpn J Oral Maxillofac Surg 2004; 50: 442-445.

DOI: 10.1038/sj.bdj.2008.1031

\section{DIABETES PROTEST}

Sir, in May 2008 the National Institute for Health and Clinical Excellence (NICE) produced a revision of the guidelines for the management of type 2 diabetes. In the guidelines there are detailed protocols for the management of the complications of diabetes, including treatment options and review intervals. There is, however, no mention of oral disease anywhere and despite the wealth of quality scientific evidence and expert opinion on the relationship between diabetes and periodontal disease, there is no recommendation in the guidelines for patients with diabetes to see a dentist!

This is not a surprise, considering that there was not a dentist on the Guidelines Development Group or on the panel of experts consulted. I don't know if anyone was asked to present a dental perspective, or were we, as a profession, ignored by our medical colleagues once again? If anyone knows, please let me know.

If readers believe that guidelines for the management of people with diabetes should include advice on the management of oral diseases and to visit a dentist then please contact: Mr Andrew Dillon, National Institute Health and Clinical Excellence, MidCity Place, 71 High Holborn, London, WC1V 6NA. Email nice@nice.org.uk DOI: 10.1038/sj.bdj.2008.1032 


\section{BISPHENOL ALERT}

Sir, a recent study examines associations between urinary Bisphenol A (BPA) concentrations and adult health status. ${ }^{1}$ The study concludes that higher BPA exposure, reflected in higher urinary concentrations of BPA, may lead to a higher incidence of disease in the adult population. The quarter of the population with the highest BPA levels were more than twice as likely to have heart disease and/ or diabetes when compared to the quarter with the lowest levels. Also, higher BPA levels were associated with abnormal liver enzyme concentrations. This paper is discussed by Saal and Myer, ${ }^{2}$ concluding that 'decreasing exposure to BPA and developing alternatives to its use are the logical next steps to minimise risk to public health'.

BPA is in widespread use in dentistry, in adhesives as BIS-GMA (bisphenol A glycidyl methacrylate) and in composite filling materials.

There are very little data about adverse events relating to BPA in dentistry, the paper by Söderholm and Mariotti ${ }^{3}$ being one of the few, which looks at the potential of these agents to mimic or disrupt oestrogenic cell responses. The overall conclusion is that there is little to worry about, but longer term effects need to be studied, together with pharmacological evaluation of dental materials.

It would appear that this is an area where further research is needed, especially as we are gradually moving away from amalgam to other materials, composite resins being an important alternative in many instances.

\section{T. M. Johnson, Tollerton}

1. Lang I A, Galloway T S, Scarlett A et al. Association of urinary bisphenol A concentration with medical disorders and laboratory abnormalities in adults. JAMA 2008; 300: 1303-1310.

2 Saal S, Myers J. Bisphenol A and risk of metabolic disorders. JAMA 2008; 300: 1353-1355.

3. Söderholm K, Mariotti A. BIS-GMA--based resins in dentistry: are they safe? J Am Dent Assoc 1999; 130: 201-209.

DOI: $10.1038 /$ sj.bdj.2008.1033

\section{FINANCIAL PLANNING}

Sir, I read with great interest the editorial In your debt (Br Dent $J$ 2008; 205: 219) and could not help but agree that financial planning is not something that most dentists worldwide are taught, whether in pre-university days or during their professional dental training. I wish to share our experience on a survey done in Malaysia about two years ago with regards to the financial knowledge and motivation to pursue a career in dentistry.

In a survey of 380 dental students with 255 responding, we found that most undertake this course out of interest, with only $16.1 \%$ for financial gain. Only one-third of them think they can become rich as a dentist. This is of course good news as it will ensure that we get suitably dedicated dentists who treat their patients not merely for monetary reward. However, we also found that their knowledge of financial planning, insurance, investment, pension planning and so forth was shallow. For example, $87 \%$ of the respondents had no idea what indemnity insurance is despite its importance to their eventual working lives.

Similar to the BDA reports most of our students will be in debt by the time they complete their course because their sponsors are usually their family, with or without scholarship or loan (81.9\%). Only $9 \%$ were fortunate to be sponsored fully by their parents. Debt in a local context may include repayment of study loan, scholarship in the form of a bond to the sponsor and repayment to the parents in the form of parental support for the future. Hence, like British and Canadian dental students, ${ }^{1}$ Malaysian students are restricted in their choice of future job options and career prospects too. Most indicate a preference to work for the private sector as opposed to the public sector with a third group unsure. This of course will limit the number of those who wish to further specialise or teach in a dental school.

We have no solution to this problem but hope improvement may be made by incorporating the teaching of financial planning at a very early stage.

W. C. Ngeow

On behalf of K. F. Choong, T. K. Ong, C. N. Shim, J. M. Wee, M. Y. Lee, C. Z. Gan and

C. W. Yeap

1. Walton J N, Matthew I R, Dumaresq C, Sudmant W. The burden of debt for Canadian dental students: part 4. The influence of debt on program and career decisions. J Can Dent Assoc 2006; 72: 913.

DOI: 10.1038/sj.bdj.2008.1034 